\title{
Isolation, Characterization and Growth Kinetic Comparison of Bone Marrow and Adipose Tissue Mesenchymal Stem Cells of Guinea Pig
}

\author{
Ghaem Aliborzi $^{1,2}$, Akbar Vahdati, ${ }^{1,2}$, Davood Mehrabani ${ }^{3}$, Seyed Ebrahim Hosseini ${ }^{1,2}$, Amin Tamadon ${ }^{3}$ \\ ${ }^{I}$ Department of Biology, Fars Science and Research Branch, Islamic Azad University, Fars, Iran \\ ${ }^{2}$ Department of Biology, Shiraz Branch, Islamic Azad University, Shiraz, Iran \\ ${ }^{3}$ Stem Cell and Transgenic Technology Research Center, Shiraz University of Medical Sciences, Shiraz, Iran
}

Background: Mesenchymal stem cells (MSCs) from different sources have different characteristics. Moreover, MSCs are not isolated and characterized in Guinea pig for animal model of cell therapy.

Aim of the Work: was the isolating of bone marrow MSCs (BM-MSCs) and adipose tissue MSCs (AT-MSCs) from Guinea pig and assessing their characteristics.

Material and Methods: In this study, bone marrow and adipose tissue were collected from three Guinea pigs and cultured and expanded through eight passages. BM-MSCs and AT-MSCs at passages 2, 5 and 8 were seeded in 24-well plates in triplicate. Cells were counted from each well $1 \sim 7$ days after seeding to determine population doubling time (PDT) and cell growth curves. Cells of passage 3 were cultured in osteogenic and adipogenic differentiation media. Results: BM-MSCs and AT-MSCs attached to the culture flask and displayed spindle-shaped morphology. Proliferation rate of AT-MSCs in the analyzed passages was more than BM-MSCs. The increase in the PDT of MSCs occurs with the increase in the number of passages. Moreover, after culture of BM-MSCs and AT-MSCs in differentiation media, the cells differentiated toward osteoblasts and adipocytes as verified by Alizarin Red staining and Oil Red O staining, respectively.

Conclusion: BM-MSCs and AT-MSCs of Guinea pig could be valuable source of multipotent stem cells for use in experimental and preclinical studies in animal models.

Keywords: Mesenchymal stem cells, Guinea pig, Adipose tissue, Bone marrow, Characterization

\footnotetext{
Accepted for publication November 25, 2015, Published online May 30, 2016

Correspondence to Davood Mehrabani

Stem Cell and Transgenic Technology Research Center, Shiraz University of Medical Sciences, Shiraz 71348-73985, Iran

Tel: +98-71-32341025, Fax: +98-71-32341025

E-mail:mehrabad@sums.ac.ir

Co-Correspondence to Amin Tamadon

Stem Cell and Transgenic Technology Research Center, Shiraz University of Medical Sciences, Shiraz 71348-73985, Iran

Tel: +98-71-32341025, Fax: +98-71-32341025

E-mail: amintamaddon@yahoo.com

(a) This is an open-access article distributed under the terms of the Creative Commons Attribution Non-Commercial License (http://creativecommons.org/ licenses/by-nc/4.0/), which permits unrestricted non-commercial use, distribution, and reproduction in any medium, provided the original work is properly cited.
} 


\section{Introduction}

In the past decade, there have been a large number of researches on mesenchymal stem cells (MSCs), reporting their biological characteristics, experimental and clinical applications (1). MSCs are recognized by two important properties of extensive self-renewal ability and multi-lineage differentiation potential into mesenchymal and nonmesenchymal cell lineages (2). During the last few years isolations of adult mesenchymal stem cells from different sources have been reported. Bone marrow-derived MSCs (BM-MSCs) were first discovered by Friedenstein et al. (3), as an adherent fibroblast-like population, are still the most frequently investigated cell type and often identified as the gold standard. However, similar cell population have been also isolated from other tissues such as liver, lung, spleen (4), dental pulp (5), umbilical cord (6), peripheral blood (7), and adipose tissue (2).

On the other hand, although bone marrow has been considered as a main source for isolation of multipotent MSCs, because of the painful isolation process and low cell yield of BM-MSCs (8), adipose tissue has recently been attracting attention as a suitable and rich source of MSCs, giving multipotent cell population, named adipose tissue derived MSCs (AT-MSCs) (9). Unlike bone marrow, adipose tissue is abundantly accessible source of stem cell that can be collected in a large volume with minimal morbidity, which therefore reduces the time in culture required to generate a therapeutic cell dose (9). AT-MSCs were first extracted and described by Radble et al. (10), who collected rat adipose tissue sections through an open surgery. During the last decade, there has been an important attempt within the scientific community to focus on the characterization of MSCs were obtained from adipose tissue compared with those from bone marrow in different species $(8,11,12)$. Even in those limited researches, there is disputation regarding in particular the growth properties of AT-MSCs versus BM-MSCs. While some studies have indicated, BM-MSCs possess a higher proliferation rate than AT-MSCs in culture media (13), the others have reported the higher significant comparative growth rate of AT-MSCs (14). Furthermore, differentiation potential as a main factor of isolated MSCs, beside of proliferation capacity and senescence of these cells are important aspect with regard to their application in cell therapy and tissue engineering (15).

MSCs derived from Guinea pig as an animal model which has several parallels with the human condition can be used in auditory, immune system, skin and joint dis- ease studies. Anatomy of the Guinea pig ear is important for inner ear studies (16). Similar immune system to human, similar antigen-macrophage interaction and delayed cutaneous hypersensitivity reaction (17) initiation and osteoarthritis progression in Guinea pig which develops spontaneously with advancing age are similar to human (18). Therefore, the goals of the current study were to isolate, culture and compare Guinea pig BM-MSCs and ATMSCs for their growth characterization, and proliferation capacity using growth curve analysis and population doubling time (PDT). Furthermore, we analyzed BM-MSCs and AT-MSCs differentiation potential to osteogenic and adipogenic cell lineages. Our observations in this study could provide some experimental evidences on choosing a suitable cell source for a particular therapeutic purpose in Guinea pig animal model.

\section{Materials and Methods}

\section{Animals}

In this experimental study, bone marrow and adipose tissue were collected from three adult male (4 months old) Guinea pigs weighing 400 450 g, which obtained from the Laboratory Animal Center of Shiraz University of Medical Science, Shiraz, Iran. All procedures are in accordance with animal guideline care of Ethical Committee of Shiraz University of Medical Sciences. All applicable institutional and national guidelines for the care and use of laboratory animals were followed. They were housed under standard conditions for a week prior to use.

\section{AT-MSCs isolation}

To establish the AT-MSCs culture, the animals were sacrificed by diethyl ether. Abdominal adipose tissue was isolated and collected in 15-ml sterile tubes. Under sterile conditions, the excised adipose tissue was rinsed with phosphate-buffered saline (PBS, Sigma-Aldrich), minced into small pieces and digested with collagenase type I (Sigma-Aldrich) at $37^{\circ} \mathrm{C}$ for 20 minutes. The samples were neutralized with an equal volume of Dulbecco Modified Eagle Medium (DMEM) supplemented with 10\% fetal bovine serum (FBS, Gibco) and centrifuged at 1,200 rpm for 7 minutes. The pellet was then filtered through a $200 \mu \mathrm{m}$ nylon mesh to remove undigested tissue. Adipose digested cells were suspended in DMEM supplemented with 10\% FBS, $1 \%$ penicillin and streptomycin (Sigma-Aldrich) and $1 \% \mathrm{~L}$-glutamine (Sigma-Aldrich) and cultured in $75-\mathrm{cm}^{2}$ flasks. The cultures were incubated at $37^{\circ} \mathrm{C}$ with $5 \% \mathrm{CO}_{2}$ and saturated humidity. The first culture media was 
changed after $24 \mathrm{~h}$ to remove non-adherent cells and the adherent cells were cultured and passaged to expand the MSCs population. The subsequent medium exchange was performed every $3 \sim 4$ days till the cultures approximately $80 \sim 90 \%$ confluence. The adherent cells were washed twice with PBS and the cells were harvested using with $0.25 \%$ trypsin (Gibco) for 2 3 min and the enzyme was inactivated with same amount of culture media. AT-MSCs were passaged up to eight times. At each passage the cells were counted and analyzed for cellular growth. Spindleshaped morphology of AT- MSCs was observed and analyzed using light microscopy at every passage.

\section{BM-MSCs isolation}

To establish the BM-MSCs culture, under sterile condition both femur and tibia from the guinea pigs were excised and carefully cleaned of adherent soft tissue. The ends of the bones were cut away and bone marrow was harvested by flushing with $10 \mathrm{ml}$ syringe with DMEM supplemented media as explained above. After washing and centrifugation at 1,200 RPM for 5 minutes, cell pellet was collected and cultured in a $75-\mathrm{cm}^{2}$ flask in a DMEM medium supplemented by $15 \%$ FBS and antibiotics. The cultures were incubated at $37^{\circ} \mathrm{C}$ in a $5 \% \mathrm{CO}_{2}$ environment and saturated humidity. After incubation, the culture media was changed and passaged as described above for AT-MSC.

\section{Reverse transcription-polymerase chain reaction (RT-PCR)}

AT-MSCs and BM-MSCs were examined for expression of surface markers using RT-PCR. Total RNA of AT-MSCs and BM-MSCs at passage 3 of the Guinea pigs were extracted according to manufacturer's instructions using RNX-Plus buffer (Cinnagen, Iran). Total RNA concentration was evaluated by spectrophotometer. After that, complementary DNA (cDNA) synthesis from DNA-free RNA (500 ng) samples was done by using Accu Power Cycle Script RT PreMix Kit (Bioneer, Korea) according to the manufacturer's protocol. Before reverse transcription, the RNA samples were digested with DNase to remove contaminating genomic DNA. Specific primers were designed based on sequences corresponding to highly conserved regions of CD90, CD45, and CD34 in Guinea pig. The primer sequences used are summarized in Table 1.

The RT-PCR amplification conditions for CD90, CD45, and $\mathrm{CD} 34$ were as followed: an initial amplification at $95^{\circ} \mathrm{C}$ for $5 \mathrm{~min}$ followed by 30 cycles of denaturation at $95^{\circ} \mathrm{C}$ for $30 \mathrm{sec}$, annealing at $64^{\circ} \mathrm{C}, 62^{\circ} \mathrm{C}$, and $61^{\circ} \mathrm{C}$ for 30 sec, respectively and extension at $72^{\circ} \mathrm{C}$ for $30 \mathrm{sec}$, with a final polymerization at $72^{\circ} \mathrm{C}$ for $5 \mathrm{~min}$. PCR Products were analyzed on $1.5 \%$ agarose gel electrophoresis and visualized by power UV light and photographed.

\section{Cell counting and growth curves}

In the present investigation, growth curves were plotted for each MSCs derived from abdominal adipose tissue and bone marrow tissues in order to better compare growth kinetics of the cells (19). For the assessment of growth characteristics, BM-MSCs and AT-MSCs at passage 2, 5 and 8 were seeded in a 24 -well plates at a density of approximately $5 \times 10^{4}$ cells per well in triplicate. Cells were collected from each well $1 \sim 7$ days after seeding and counted microscopically to produce cell growth curves. The curves were drawn using GraphPad Prism (Version 5.01; GraphPad software Inc., San Diego, CA, USA).

\section{Calculation of PDT}

To compare the in-vitro proliferation rate, the PDT value was determined for each studied cells. PDT was calculated using the formula $\mathrm{PDT}=\mathrm{T} \ln 2 / \ln (\mathrm{Xe} / \mathrm{Xb})$, in which $\mathrm{T}$ is the incubation time in hours, $\mathrm{Xb}$ represents the cell number at the beginning of the incubation time and $\mathrm{Xe}$ corresponds to the cell number at the end of incubation time.

\section{Osteogenic and adipogenic differentiation assay}

In order to compare the differentiation potential of

Table 1. The primers used for RT-PCR analysis of mesenchymal cell confirmation of adipose tissue and bone marrow derived mesenchymal stem cells in Guinea pig

\begin{tabular}{cllc}
\hline Gene name & Direction & \multicolumn{1}{c}{ Primer sequence } & Amplicon length (bp) \\
\hline CD34 & Forward & CAC CAC GGT ATT CAC CAC CA & 484 \\
& Reverse & GTG GAT CCC CAG CTT TTC CA & 328 \\
CD44 & Forward & ACC TGC AGT TTG CAT TGC TG & 281 \\
& Reverse & ACG TGG AAC ACA CCT GCA TA & \\
CD90 & Forward & TGC TGG CAG TCT TAC AGG T & \\
& Reverse & TGT GTA GGT CCC TTC GTC CT
\end{tabular}


BM-MSCs and AT-MSCs, cells of passage 3 were used and osteogenic and adipogenic differentiation were induced. For osteogenic differentiation BM-MSCs and AT-MSCs were seeded in 6-well plates. After the cells reaching 70\% confluency, they were cultured for 3 weeks in osteogenic medium containing low glucose DMEM supplement with $100 \mathrm{nM}$ dexamethasone (Sigma-Aldrich), $0.05 \mathrm{mM}$ ascorbate-2-phosphate (Wako Chemicals, Richmond, VA, USA), $10 \mathrm{mM}$ b-glycerophosphate (Sigma-Aldrich), 1\% antibiotic/antimycotic and 10\% FBS. The medium was replaced every 3 days. At day 21, the cells were fixed by $10 \%$ formalin solution (Sigma-Aldrich), and then stained using Alizarin Red (Sigma-Aldrich) to detect calcified extracellular matrix and osteogenic differentiation.

For adipogenic differentiation BM-MSCs and AT-MSCs were seeded in 6-well plates. When they reached 70\% confluency, were induced to adipogenic differentiation with adipogenic induction medium containing DMEM low glucose, 10\% FBS, $0.5 \mathrm{mM}$ isobutyl-methylxanthine (SigmaAldrich), 10\% FBS, $0.5 \mathrm{mM}$ isobutyl-methylxanthine (Sigma-Aldrich), $1 \mu \mathrm{M}$ dexamethasone, $10 \mu \mathrm{M}$ insulin, $200 \mu \mathrm{M}$ indomethacin (Sigma-Aldrich). The plates were maintained for three weeks and medium was replaced every $3 \sim 4$ days. At the end of period, the cultures were fixed by $10 \%$ formalin solution for 10 minutes. Fixed cells were subjected to Oil Red O (Sigma-Aldrich), which specifically stains lipid droplets.

\section{Statistical analysis}

The mean and SE of counted cells in growth curve analysis were compared using one-way ANOVA (SPSS for Windows, version 11.5, SPSS Inc, Chicago, USA) and Tukey post-hoc test. Values of $\mathrm{p} \leq 0.05$ were considered significant.

\section{Results}

\section{Phenotypic characterization of the cells}

BM-MSCs and AT-MSCs were isolated from Guinea pig. After 48 hours, cells attached to the base of the tissue culture flask. The number of round-shaped cells gradually decreased and the growth rate of the fibroblastic cells gradually increased in culture media. Eight successive passages were done after reaching 70 80\% confluency each time. Fibroblast-like cells were observed in all passages (Fig. 1).

\section{Cell surface markers of AT-MSCs and BM-MSCs}

The expressions of cell surface markers were shown in AT-MSCs and BM-MSCs by RT-PCR analysis (Fig. 2). Both AT-MSCs and BM-MSCs were positive for MSC markers (CD44 and CD90) and negative for hematopoietic markers (CD34).

\section{Growth Characteristics of the MSCs}

According to our result the PDT of the passages 2, 5, and 8 of the AT-MSCs were $59.7 \mathrm{~h}, 64.2 \mathrm{~h}$ and $80.9 \mathrm{~h}$,
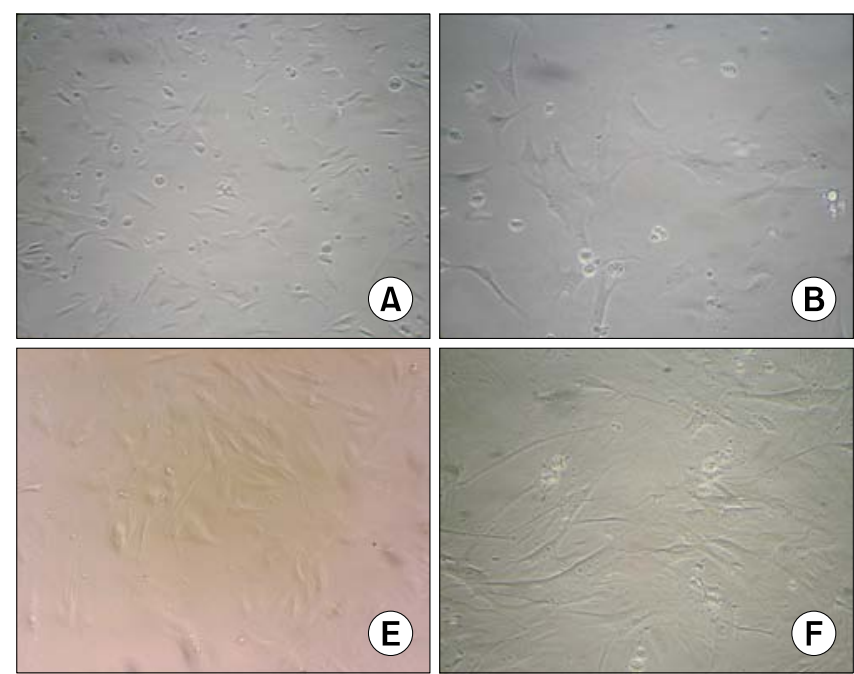

B)

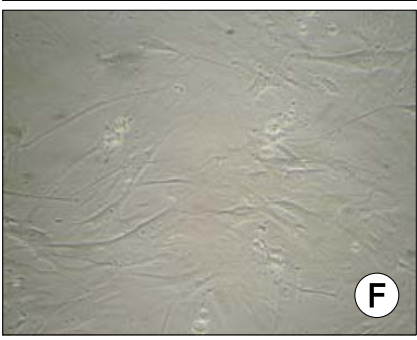

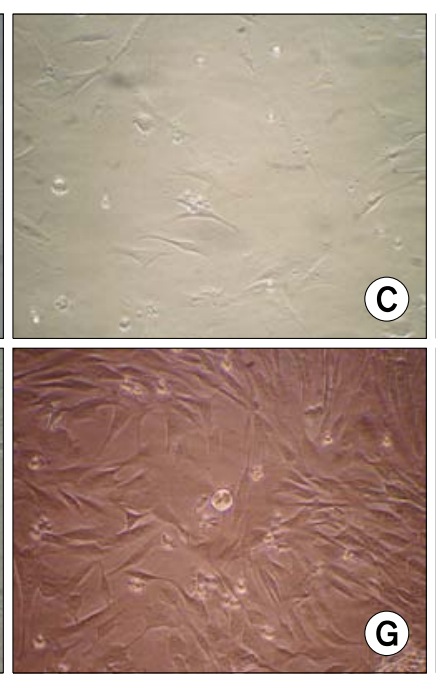

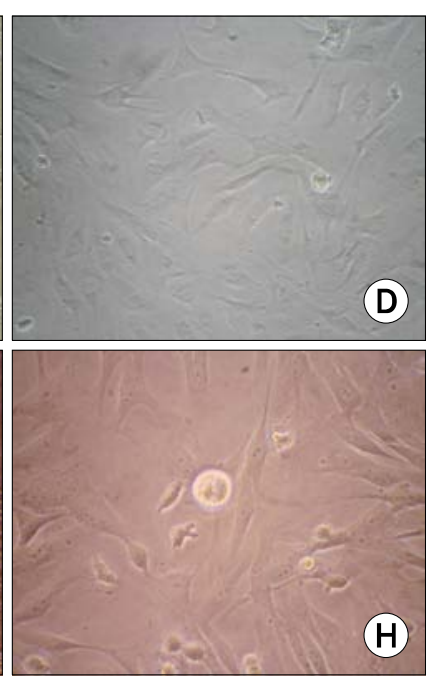

Fig. 1. Morphologic characteristics of adipose tissue-derived and bone marrow-derived mesenchymal stem cells (AT-MSCs and BM-MSCs, respectively) of Guinea pig. Most MSCs showed fibroblastic morphology regardless of the cell source. (A) Primary culture of AT-MSCs $(\times 40)$, (B) Passage 2 of AT-MSCs $(\times 100)$, (C) passage 5 of AT-MSCs $(\times 100)$, (D) passage 8 of AT-MSCs $(\times 100)$, (E) Primary culture of BM-MSCs $(\times 100)$, (F) passage 2 of BM-MSCs $(\times 100)$, (G) passage 5 of BM-MSCs $(\times 100)$, and $(H)$ passage 8 of BM-MSCs $(\times 200)$. 
respectively. Also PDT was $62.9 \mathrm{~h}, 65.6 \mathrm{~h}$ and $91.4 \mathrm{~h}$ at the passages 2, 5, and 8 for BM-MSCs. Both AT-MSCs and BM-MSCs showed enough good proliferation rates in pas- sages 2, 5, and 8, especially at passages 2 and 8. By comparing of growth curves, proliferation rate of AT-MSCs was more than BM-MSCs in passages 2 (Fig. 3A), 5 (Fig.
A

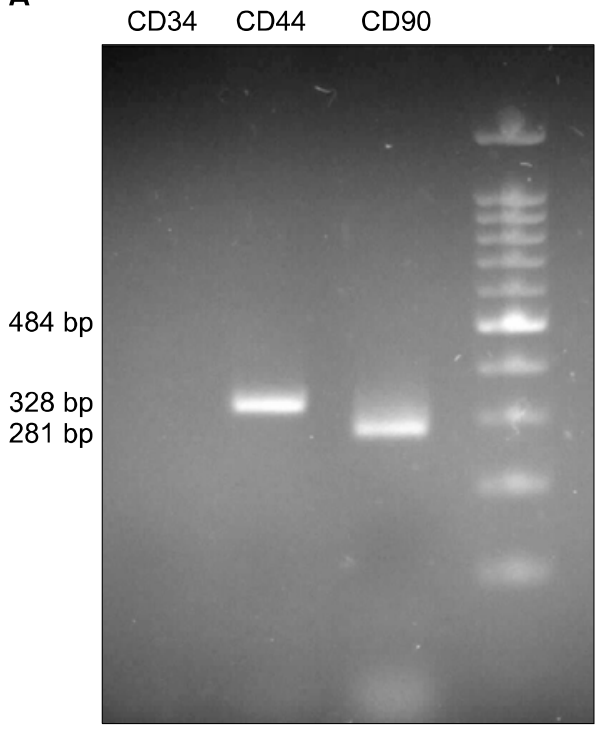

B

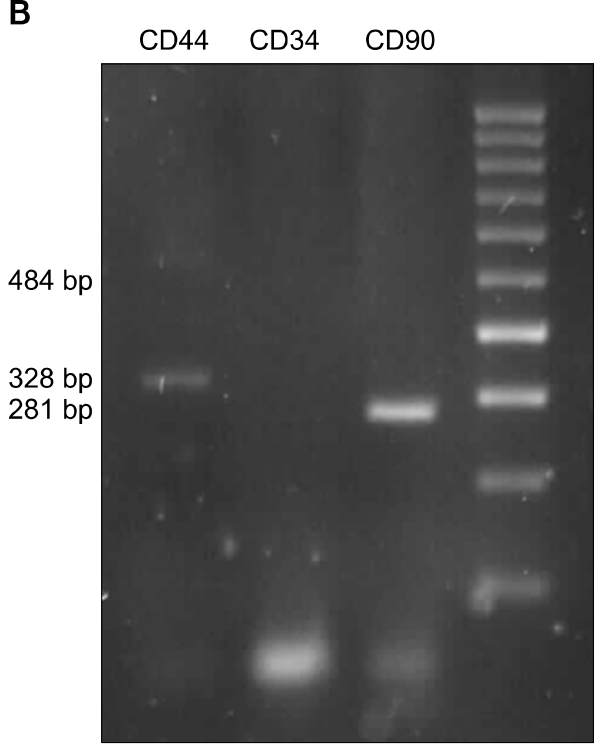

Fig. 2. Agarose gel electrophoresis of (A) bone marrow and (B) adipose tissue-derived mesenchymal stem cells RT-PCR products show positive expression for CD44 and CD90 (mesenchymal surface marker) and negative expression for CD34 (hematopoietic surface marker).
A

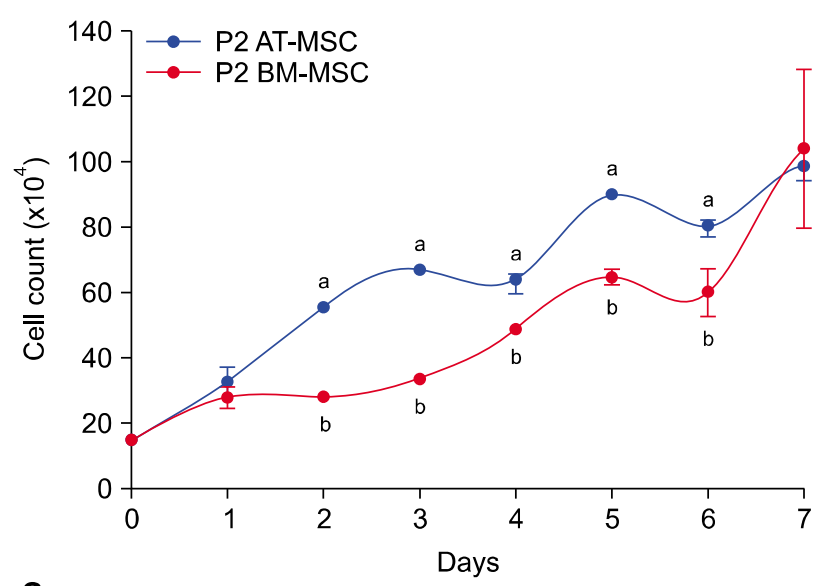

C

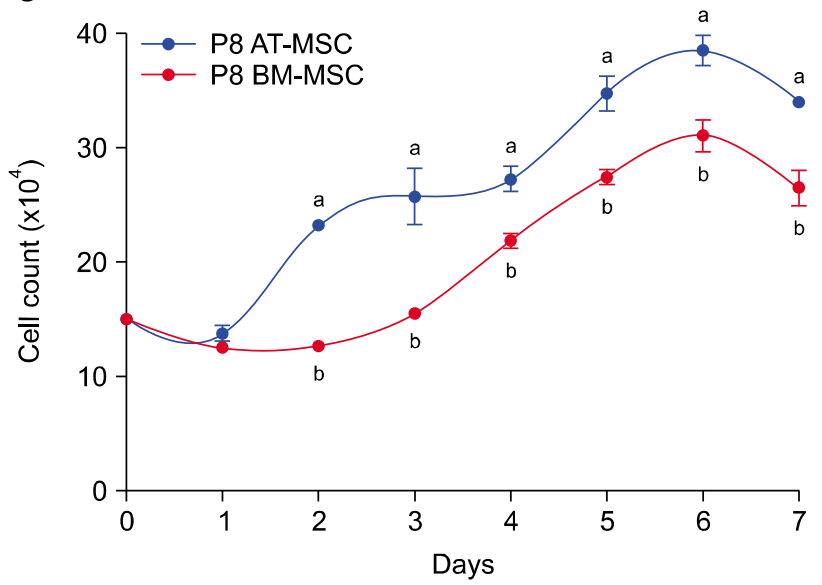

B

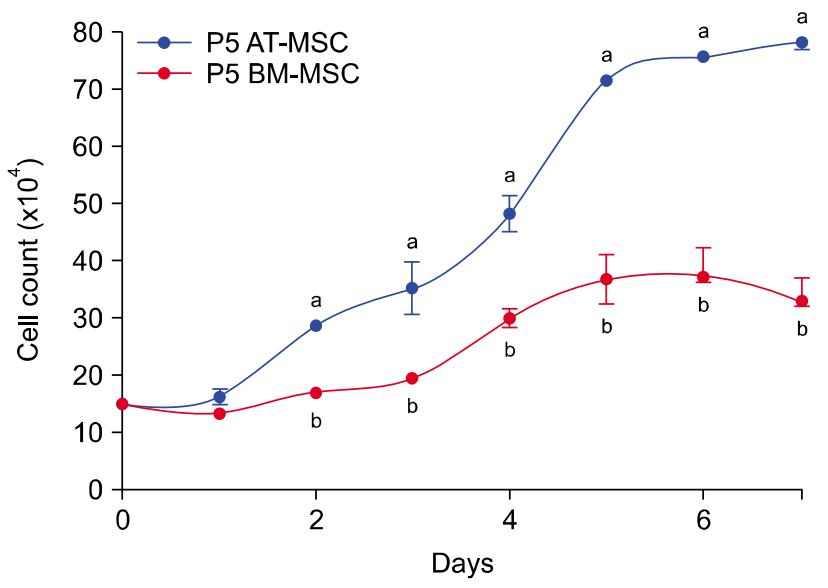

Fig. 3. Comparison of mean and standard error of cell counts between growth curves of guinea pig bone marrow-derived mesenchymal stem cells (BM-MSCs) and adipose tissue-derived mesenchymal stem cells (AT-MSCs) at passages 2, 5 and 8. Proliferation rate of AT-MSCs was more than BM-MSCs, (A) in passages 2, (B) in passages 5 , and $(C)$ in passages $8 .{ }^{a, b}$ Superscript letters show differences between the number of cells in each curve in the same day $(p<0.05)$. 
A

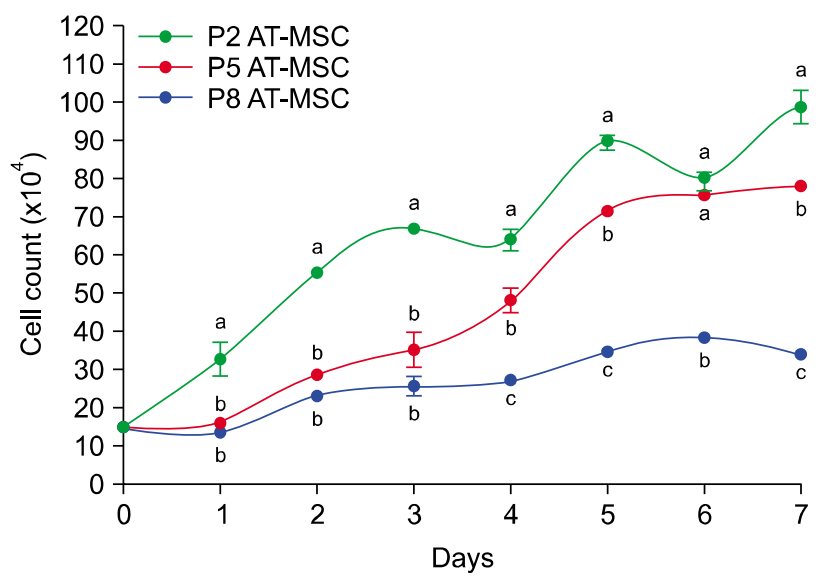

B

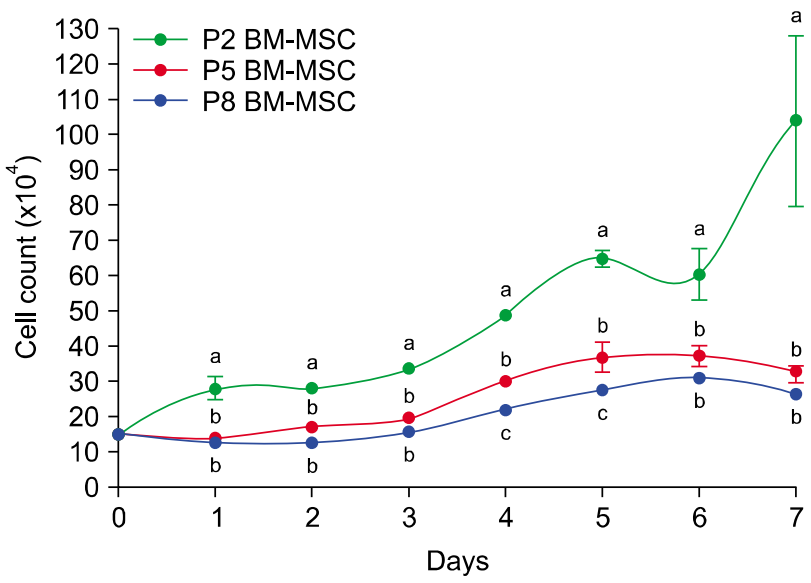

Fig. 4. Comparison of mean and standard error of cell counts in growth curves of guinea pig bone marrow-derived mesenchymal stem cells (BM-MSCs) and adipose tissue-derived mesenchymal stem cells (AT-MSCs) between passages 2, 5 and 8. The proliferation rate of AT-MSCs (A) and BM-MSCs (B) decreased gradually from passage 2 to passage $8 .{ }^{\mathrm{a}, \mathrm{b}, \mathrm{c}}$ Superscript letters show differences between the number of cells in each curve in the same day $(p<0.05)$.
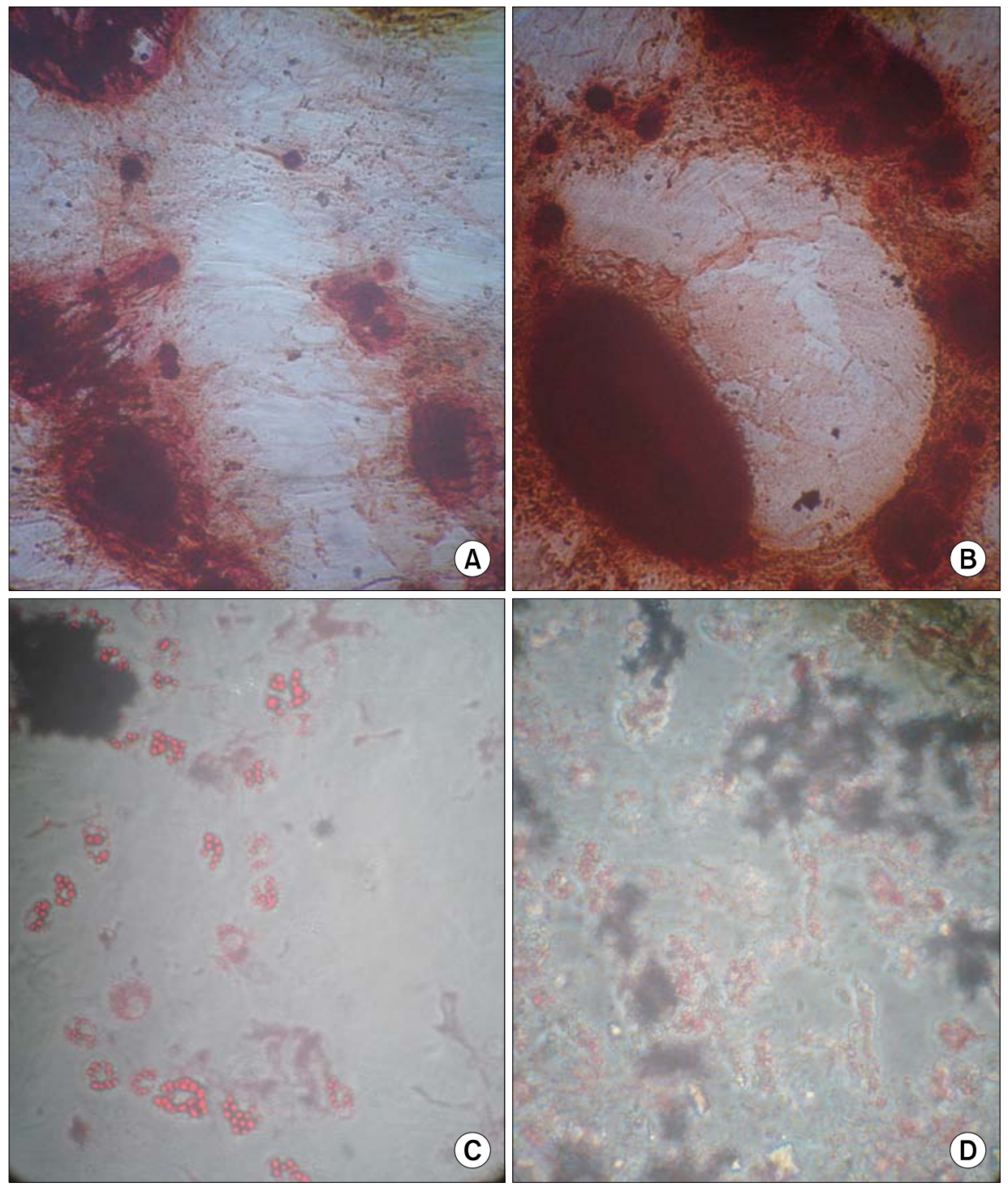

Fig. 5. Osteogenic and adipogenic differentiations of bone marrow-derived (A and $\mathrm{C}$, respectively) and adipose tissue-derived ( $B$ and $D$, respectively) mesenchymal stem cells (BM-MSCs and AT-MSCs) from passage 3 of subcultures by Alizarin Red staining and Oil Red staining, respectively. 
3B), and 8 (Fig. 3C; $\mathrm{p}<0.05$ ). In this study the proliferation rate of AT-MSCs (Fig. 4A) and BM-MSCs (Fig. 4B) decreased gradually from passage 2 to passage 8 .

\section{Differentiation assay}

Both types of MSCs successfully differentiated into adipocytes and osteoblasts lineages (Fig. 5). The negative control (non-induced) cells for each type of differentiation were negative for Alizarin Red and Oil Red $\mathrm{O}$ stains. Osteogenesis of BM-MSCs and AT-MSCs demonstrates mineralized matrix 21 days after induction. Adipogenesis of BM-MSCs and AT-MSCs as detected by the formation of lipid droplets stained with Oil Red O staining, 21 days after induction.

\section{Discussion}

In addition to bone marrow, as a main source of MSCs, adipose tissue has been known as a source of MSCs (14). Moreover, abdominal adipose tissue is abundantly accessible and often applied for isolation of MSCs and tissue engineering (20). Therefore, some previous researches compared MSCs from adipose tissue with those from bone marrow but the results concerning comparative growth properties and differentiation potential of the cells remained as the subject of controversy $(14,21)$. Since, in the present research, Guinea pig AT-MSCs of abdominal regions as well as BM-MSCs were isolated and compared in terms of their in-vitro morphology, growth characteristics and differentiation capacity. Isolated Guinea pig AT-MSCs and BM-MSCs had plastic adherence capability and expanded in-vitro. These cells exhibited typical fibroblastic-like morphology, as one of MSCs recognition parameters, in all passages. To further characterize these cells, cell surface markers were examined by using RTPCR. MSCs from both sources displayed, presence of mesenchymal cell surface markers (CD44 and CD90) and the lack of hematopoietic cell surface marker (CD34).

According to the growth curve analysis, growth and proliferation rate of AT-MSCs and BM-MSCs decreased gradually from passage 2 to passage 8 . Consistent with our results, with increasing passage number of the human MSC, proliferation, growth rate and the number of cells in culture were decreased $(22,23)$. By comparing of growth curves, growth and proliferation rate of AT-MSCs was more than BM-MSCs, especially in passage 8 in Guinea pigs. Also, in the rat, AT-MSCs possessed a higher expansion rate than BM-MSCs (15). Moreover, in human, AT-MSCs have also been shown to have higher proliferation capacities than BM-MSC (24). The PDT of Guinea pig BM-MSCs in analyzed passages 2, 5, and 8 were more than AT-MSCs. Moreover, in the present study it was observed that BM-MSCs showed a bit increase in size versus AT-MSCs. The same as our findings, Peng et al. (14) described PDT of $45.2 \mathrm{~h}$ for AT-MSC and $61.2 \mathrm{~h}$ for BMMSC in human. Moreover, they revealed that the human BM-MSC were morphologically larger than AT-MSC (14). Therefore, BM-MSCs density can be rapidly increased in culture and reached saturation density earlier than ATMSCs. Hyper-density of cell population displayed a negative role against cell proliferation because of contact inhibition and reciprocal effects of cells on each other (14, 25). AT-MSCs seemed to have less percentage of senescent cells and more proliferation rate during the passages in culture media than the BM-MSCs (15). It should however be noted that the origin of the cells, the cultivation conditions and various medium supplements may have an effect on PDT of MSCs (26). Wagner et al. (27) analyzed global gene expression profiles of human MSCs isolated from adipose tissue versus bone marrow; who demonstrated that higher proliferation capacity in AT-MSCs could be due to significant differences in the gene expression patterns and higher expressed of cell division cycle associated 8 (CDCA8), and cyclin B2 (CCNB2) gens in AT-MSC than in BM-MSC.

In addition to proliferation potential, differentiation capacity has been considered as an important quality of MSCs. In this experimental study, to assess the differentiation potential of AT-MSCs and BM-MSCs, passage 3 cells were used. Differentiation is a process which dramatically changes a cell in size, morphology, membrane potential and metabolic activity caused by highly-controlled modifications in gene expression (28). According to Alizarin Red staining for mineralized matrix in osteogenic differentiation and Oil Red staining for lipid droplets in osteogenic differentiation, AT-MSCs and BM-MSCs differentiated into adipose and bone cell lineage similar to previous studies $(29,30)$. Although various differentiation ability in MSCs that reported in some previous research could be due to, different genes expressed in MSCs from different ontogenetic sources and culture conditions (27).

\section{Conclusion}

Despite the importance of Guinea pig as an experimental animal model for many conditions, the characterization of Guinea pig MSCs is still limited. In this study, we have successfully isolated and compared MSCs population from Guinea pig adipose and marrow tissue. ATMSCs and BM-MSCs sources are highly accessible, me- 
chanically stable and readily expanded in culture flask. These cells had enough good proliferation capacity and display high potential toward adipogenic and osteogenic differentiation, while proliferation rate and PDT of ATMSCs were better than the same cell derived from BMMSCs. The resent study results showed that although adipose tissue may prove to be a more efficient source of MSC and there were some differences between BM-MSCs and AT-MSCs, but these cell sources can be considered as an appropriate candidate for use in preclinical studies and regenerative medicine applications.

\section{Acknowledgments}

We thank Dr. Mehdi Dianatpour and Dr. Zahra Khodabandeh for valuable advice and Dr. Iman Razeghian Jahromi and Mr. Shahrokh Zare for technical support to this study. Authors wish thanks the technical and equipment supports of Stem Cell and Transgenic Technology Research Center, Shiraz University of Medical Sciences, and the official and funding support of Shiraz Branch, Islamic Azad University, and Fars Science and Research Branch, Islamic Azad University, Fars, Shiraz, Iran. This study was funded by Islamic Azad University, Shiraz Branch, Shiraz, Iran (grant no. 91.15007; date 19/1/2013).

\section{Potential conflict of interest}

The authors have no conflicting financial interest.

\section{References}

1. Wang S, Qu X, Zhao RC. Clinical applications of mesenchymal stem cells. J Hematol Oncol 2012;5:19

2. Mehrabani D, Hassanshahi MA, Tamadon A, Zare S, Keshavarz S, Rahmanifar F, Dianatpour M, Khodabandeh Z, Jahromi I, Tanideh N, Ramzi M, Aqababa H, Kuhi-Hoseinabadi O. Adipose tissue-derived mesenchymal stem cells repair germinal cells of seminiferous tubules of busulfan-induced azoospermic rats. J Hum Reprod Sci 2015;8:103-110

3. Friedenstein AJ, Gorskaja JF, Kulagina NN. Fibroblast precursors in normal and irradiated mouse hematopoietic organs. Exp Hematol 1976;4:267-274

4. in 't Anker PS, Noort WA, Scherjon SA, Kleijburg-van der Keur C, Kruisselbrink AB, van Bezooijen RL, Beekhuizen W, Willemze R, Kanhai HH, Fibbe WE. Mesenchymal stem cells in human second-trimester bone marrow, liver, lung, and spleen exhibit a similar immunophenotype but a heterogeneous multilineage differentiation potential. Haematologica 2003;88:845-852

5. Mahdiyar P, Zare S, Robati R, Dianatpour M, Torabi K, Tamadon A, Razeghian Jahromi I, Tamadon A, Mehrabani D. Isolation, culture, and characterization of human dental pulp mesenchymal stem cells. Int J Pediatr 2014;2:44

6. Ding DC, Chang YH, Shyu WC, Lin SZ. Human umbilical cord mesenchymal stem cells: a new era for stem cell therapy. Cell Transplant 2015;24:339-347

7. Anasetti C, Logan BR, Lee SJ, Waller EK, Weisdorf DJ, Wingard JR, Cutler CS, Westervelt P, Woolfrey A, Couban S, Ehninger G, Johnston L, Maziarz RT, Pulsipher MA, Porter DL, Mineishi S, McCarty JM, Khan SP, Anderlini P, Bensinger WI, Leitman SF, Rowley SD, Bredeson C, Carter SL, Horowitz MM, Confer DL; Blood and Marrow Transplant Clinical Trials Network. Peripheral-blood stem cells versus bone marrow from unrelated donors. N Engl J Med 2012;367:1487-1496

8. Kisiel AH, McDuffee LA, Masaoud E, Bailey TR, Esparza Gonzalez BP, Nino-Fong R. Isolation, characterization, and in vitro proliferation of canine mesenchymal stem cells derived from bone marrow, adipose tissue, muscle, and periosteum. Am J Vet Res 2012;73:1305-1317

9. Baer PC, Geiger H. Adipose-derived mesenchymal stromal/stem cells: tissue localization, characterization, and heterogeneity. Stem Cells Int 2012;2012:812693

10. Rodbell M. The metabolism of isolated fat cells. IV. Regulation of release of protein by lipolytic hormones and insulin. J Biol Chem 1966;241:3909-3917

11. Asadi-Yousefabad SL, Khodakaram-Tafti A, Dianatpour M, Mehrabani D, Zare S, Tamadon A, Nikeghbalian S, Raayat-Jahromi A, Ahmadlou S. Genetic evaluation of bone marrow-derived mesenchymal stem cells by a modified karyotyping method. Comp Clin Pathol 2015;24:1361-1366

12. Al-Nbaheen $M$, Vishnubalaji R, Ali D, Bouslimi A, Al-Jassir F, Megges M, Prigione A, Adjaye J, Kassem M, Aldahmash A. Human stromal (mesenchymal) stem cells from bone marrow, adipose tissue and skin exhibit differences in molecular phenotype and differentiation potential. Stem Cell Rev 2013;9:32-43

13. Izadpanah R, Trygg C, Patel B, Kriedt C, Dufour J, Gimble JM, Bunnell BA. Biologic properties of mesenchymal stem cells derived from bone marrow and adipose tissue. J Cell Biochem 2006;99:1285-1297

14. Peng L, Jia Z, Yin X, Zhang X, Liu Y, Chen P, Ma K, Zhou C. Comparative analysis of mesenchymal stem cells from bone marrow, cartilage, and adipose tissue. Stem Cells Dev 2008;17:761-773

15. Baghaban Eslaminejad M, Mardpour S, Ebrahimi $M$. Growth kinetics and in vitro aging of mesenchymal stem cells isolated from rat adipose versus bone marrow tissues. Iran J Vet Surg 2008;3:9-20

16. Barros BB, Andrade JS, Garcia LB, Pifaia GR, Cruz OL, Onishi ET, Penido Nde O. Micro-endoscopic ear anatomy of guinea pig applied to experimental surgery. Acta Cir Bras 2014;29 Suppl 1:7-11

17. Eyanagi R, Toda A, Imoto M, Uchiyama H, Ishii Y, Kuroki H, Kuramoto Y, Soeda S, Shimeno H. Covalent binding of nitroso-sulfonamides to glutathione S-transferase in guinea pigs with delayed type hypersensitivity. Int Immunopharmacol 2012;12:694-700 
18. Tonge DP, Bardsley RG, Parr T, Maciewicz RA, Jones SW. Evidence of changes to skeletal muscle contractile properties during the initiation of disease in the ageing guinea pig model of osteoarthritis. Longev Healthspan 2013;2:15

19. Mehrabani D, Mahboobi R, Dianatpour M, Zare S, Tamadon A, Hosseini SE. Establishment, culture, and characterization of Guinea pig fetal fibroblast cell. Vet Med Int 2014;2014:510328

20. Kakudo N, Morimoto N, Ogawa T, Kusumoto K. Potential of adipose-derived stem cells for regeneration medicine: clinical application and usefulness of fat grafting. J Stem Cell Res Ther 2014;4:204

21. Yoshimura H, Muneta T, Nimura A, Yokoyama A, Koga $\mathrm{H}$, Sekiya I. Comparison of rat mesenchymal stem cells derived from bone marrow, synovium, periosteum, adipose tissue, and muscle. Cell Tissue Res 2007;327:449-462

22. Bruder SP, Jaiswal N, Haynesworth SE. Growth kinetics, self-renewal, and the osteogenic potential of purified human mesenchymal stem cells during extensive subcultivation and following cryopreservation. J Cell Biochem 1997;64:278-294

23. Kang TJ, Yeom JE, Lee HJ, Rho SH, Han H, Chae GT. Growth kinetics of human mesenchymal stem cells from bone marrow and umbilical cord blood. Acta Haematol 2004;112:230-233

24. Kern S, Eichler H, Stoeve J, Klüter H, Bieback K. Comparative analysis of mesenchymal stem cells from bone marrow, umbilical cord blood, or adipose tissue. Stem Cells
2006;24:1294-1301

25. Suchanek J, Soukup T, Visek B, Ivancakova R, Kucerova L, Mokry J. Dental pulp stem cells and their characterization. Biomed Pap Med Fac Univ Palacky Olomouc Czech Repub 2009;153:31-35

26. Schipper BM, Marra KG, Zhang W, Donnenberg AD, Rubin JP. Regional anatomic and age effects on cell function of human adipose-derived stem cells. Ann Plast Surg 2008;60:538-544

27. Wagner W, Wein F, Seckinger A, Frankhauser M, Wirkner U, Krause U, Blake J, Schwager C, Eckstein V, Ansorge W, Ho AD. Comparative characteristics of mesenchymal stem cells from human bone marrow, adipose tissue, and umbilical cord blood. Exp Hematol 2005;33:1402-1416

28. Alipour F, Parham A, Kazemi Mehrjerdi H, Dehghani H. Equine adipose-derived mesenchymal stem cells: phenotype and growth characteristics, gene expression profile and differentiation potentials. Cell J 2015;16:456-465

29. Colleoni S, Bottani E, Tessaro I, Mari G, Merlo B, Romagnoli N, Spadari A, Galli C, Lazzari G. Isolation, growth and differentiation of equine mesenchymal stem cells: effect of donor, source, amount of tissue and supplementation with basic fibroblast growth factor. Vet Res Commun 2009;33:811-821

30. Pascucci L, Mercati F, Marini C, Ceccarelli P, Dall'Aglio C, Pedini V, Gargiulo AM. Ultrastructural morphology of equine adipose-derived mesenchymal stem cells. Histol Histopathol 2010;25:1277-1285 